\title{
Electron bunch compression with an optical laser
}

\author{
Takashi Tanaka* \\ RIKEN SPring-8 Center, Koto 1-1-1, Sayo, Hyogo 679-5148, Japan
}

(Received 28 September 2019; published 18 November 2019)

\begin{abstract}
We propose a simple scheme to compress an electron beam with an optical laser, which can potentially achieve a bunch length much shorter than what is currently available with a conventional radio-frequency technique. Instead of an energy chirp, the optical laser induces an energy modulation whose amplitude linearly changes along the longitudinal axis, which effectively compresses the electron beam after it passes through an optimized magnetic chicane. Numerical simulations performed to demonstrate the proposed scheme show that a free electron laser pulse with the peak power of $16 \mathrm{GW}$ and the pulse length of 470 as (attosecond) can be generated, if this scheme is applied to a $2-\mathrm{GeV}$ and $2-\mathrm{kA}$ electron beam.
\end{abstract}

DOI: 10.1103/PhysRevAccelBeams.22.110704

\section{INTRODUCTION}

Electron bunch compression, which refers to a process to compress an electron beam in the longitudinal direction and enhance its peak current, is a key technology in most accelerator applications such as free electron lasers (FELs). In a conventional bunch-compression process, a radiofrequency (rf) accelerator cavity induces an energy chirp on the electron beam, or generates an energy variation correlated with the longitudinal position. After passing through a magnetic chicane, higher-energy electrons move forward, while lower-energy ones move backward, and thus the electron beam is strongly compressed if the longitudinal dispersion of the chicane is optimized.

It is obvious that the bunch compression factor depends on the chirp rate induced on the electron beam, which is determined by the amplitude and frequency of the rf wave; a higher amplitude and a higher frequency result in a larger energy chirp to realize a higher compression factor, which results in a higher peak current and a shorter bunch length. To improve the compression factor, the so-called harmonic cavity is usually used in addition to the main rf cavity for acceleration, which has a higher frequency and linearizes the energy chirp. In the x-ray FEL facilities currently under operation [1-7], the rf frequency of the harmonic cavity for the bunch compression ranges from $3.9 \mathrm{GHz}$ to $12 \mathrm{GHz}$ [8$10]$, and the maximum peak current of $\sim 10 \mathrm{kA}$ and the shortest bunch length of $\sim 10$ fs or shorter are achieved by carefully tuning the accelerator parameters [11,12].

\footnotetext{
"ztanaka@spring8.or.jp
}

Published by the American Physical Society under the terms of the Creative Commons Attribution 4.0 International license. Further distribution of this work must maintain attribution to the author(s) and the published article's title, journal citation, and DOI.
To further shorten the bunch length, we need to shift the spectral band of the electromagnetic (EM) wave for the bunch compression toward higher frequencies. It should be noted, however, that a higher-frequency EM wave generated by a conventional if source is no more suitable to drive the accelerator cavity, because of a number of issues such as the rf breakdown and pulse heating [13-15]. Instead, the so-called terahertz $(\mathrm{THz})$ accelerator, which is driven by an intense $\mathrm{THz}$ radiation pulse generated by an optical laser, has been under development [16-18]; because of a much higher frequency and a much shorter pulse length, the accelerator cavity driven by the laser-based $\mathrm{THz}$ radiation does not suffer from the above issues, and has a potential to reduce the bunch length down to a level that cannot be achieved with a conventional scheme.

One disadvantage of using the laser-based $\mathrm{THz}$ radiation as an accelerator driver is the low energy-conversion efficiency (a few percent) from the optical laser to the $\mathrm{THz}$ radiation. As a result, its application to the bunch compression is currently limited to a low-energy electron beam. If the intense optical laser is used to directly induce an energy chirp on the electron beam, the bunch compression can be more efficient and the resultant bunch length can be much shorter than what is currently achieved. The purpose of this paper is to propose such a scheme, which is easily implemented in existing facilities.

\section{PRINCIPLE OF OPERATION}

First, let us recall that a sort of bunch compression with an optical laser actually takes place in seeded FELs, especially in high gain harmonic generation (HGHG) FELs [19]. In the HGHG FELs, a seed laser modulates the electrons in energy with the period of its wavelength $(\lambda)$ in an undulator called a modulator. After the electron beam passes through a magnetic chicane located downstream of the modulator, the energy modulation is converted to a 
density modulation, and the electron beam forms a train of local bunches regularly arranged with the interval of $\lambda$. Note that these local bunches are usually referred to as microbunches, each of which is much shorter than $\lambda$, if the amplitude of energy modulation is large enough. In other words, the electron beam is sliced into a number of microbunches that are significantly compressed.

Let us consider how the microbunches are formed in the HGHG FELs. To facilitate the following discussion, we introduce a relative coordinate $s=z-\bar{v}_{z} t$, where $z$ denotes the longitudinal coordinate along which the electron beam travels, $t$ denotes time, and $\bar{v}_{z}$ denotes the average velocity of the electron beam along the $z$ axis. We also define a relative energy $\eta=\left(\gamma-\gamma_{0}\right) / \gamma$, where $\gamma$ denotes the Lorentz factor of an electron and $\gamma_{0}$ is the ensemble average.

We assume that the electron beam is modulated in energy with an amplitude of $\eta_{0}$, and passes through a magnetic chicane with a longitudinal dispersion of $R_{56}$. Then an electron initially positioned at $\left(s^{\prime}, \eta^{\prime}\right)$ in the phase space moves according to

$$
\begin{gathered}
s=s^{\prime}+R_{56} \eta, \\
\eta=\eta^{\prime}+\eta_{0} \sin k s^{\prime},
\end{gathered}
$$

with $k=2 \pi / \lambda$ being the wave number of the seed laser. The optimum dispersion $\rho$ to maximize the microbunch compression factor is given as

$$
\rho \equiv \frac{\lambda}{2 \pi \eta_{0}}
$$

in which case the condition $d s / d \eta=0$ is satisfied for the initial relative coordinate $s^{\prime}=(n+1 / 2) \lambda$, where $n$ is an integer. Let us define a normalized dispersion $\hat{r} \equiv R_{56} / \rho$.

The electron distributions in the phase space under the above conditions are plotted in dashed lines in Figs. 1(a)-1(d), for different values of (a) $\hat{r}=0$, (b) $\hat{r}=1 / 2$, (c) $\hat{r}=1$, and (d) $\hat{r}=10 \pi$, assuming that the electron beam is initially cold $\left(\eta^{\prime}=0\right)$. In the same figures, current profiles $j(s)$ evaluated by integrating the distribution function

$$
f(s, \eta)=\delta\left[\eta-\eta_{0} \sin \left(k s-k R_{56} \eta\right)\right],
$$

are also plotted, where $\delta$ is the delta function. Note that the relative coordinate and energy are normalized by $\lambda$ and $\eta_{0}$, and the current profile is normalized by the maximum value $j_{\max }$ for each condition. As expected, the microbunches are compressed most strongly in the condition $\hat{r}=1$ (c).

It is worth mentioning that even under the overcompressed condition $\hat{r} \gg 1$, the microbunches survive as shown in Fig. 1(d). This is understood by recalling that the condition $d s / d \eta=0$ is satisfied for electrons with the initial relative coordinate

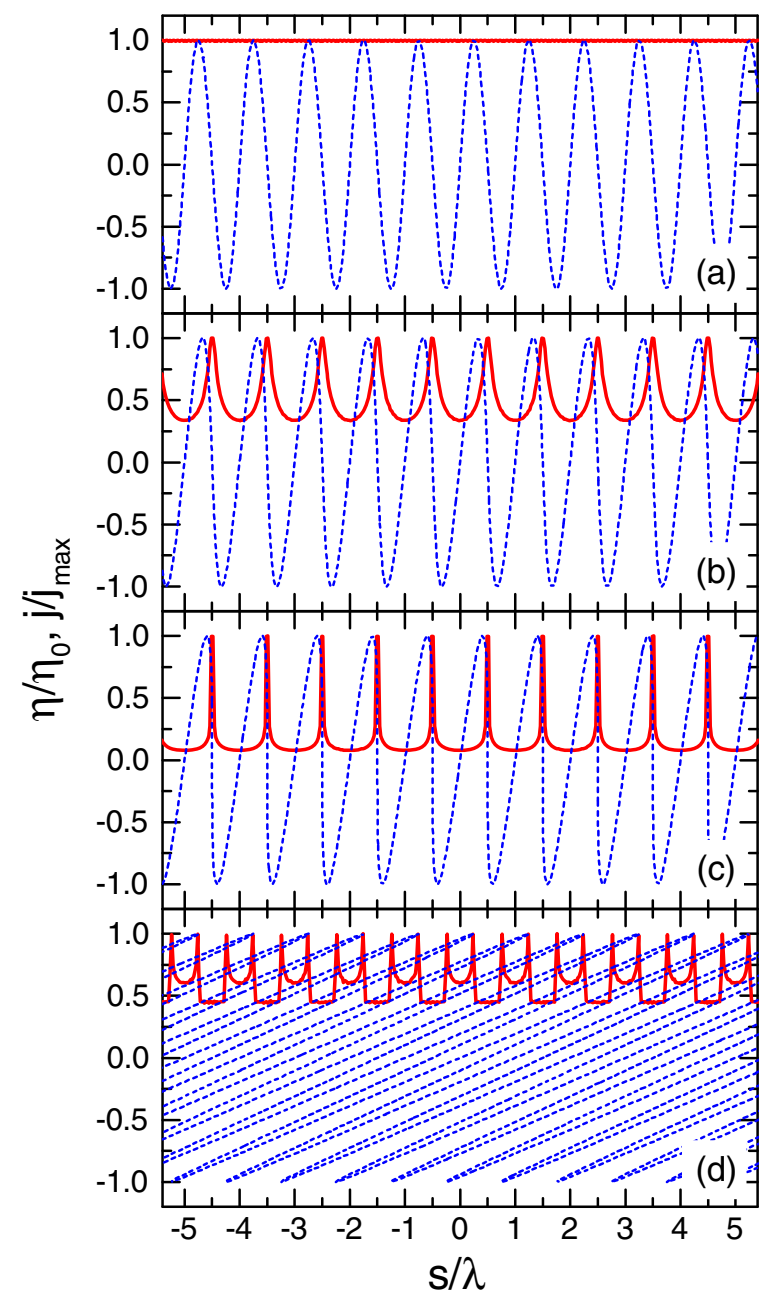

FIG. 1. Electron distributions (dashed lines) and current profiles (solid lines) in an energy-modulated electron beam for different values of the normalized dispersion $\hat{r}$ : (a) 0 , (b) $1 / 2$, (c) 1 , and (d) $10 \pi$.

$$
s^{\prime}=n \lambda \pm\left[(2 \pi \hat{r})^{-1}+\frac{1}{4}\right] \lambda,
$$

which means that two microbunches are created for each cycle of the seed laser. To be distinguished from the normal microbunches generated under the optimized condition $(\hat{r}=1)$, they are hereafter referred to as secondary microbunches.

Assuming $\hat{r} \gg 1$, the motion of the secondary microbunches in the phase space is given as

$$
\begin{gathered}
s \sim s^{\prime} \pm R_{56} \eta_{0}, \\
\eta \sim \pm \eta_{0} .
\end{gathered}
$$

This means that the secondary microbunches are formed by electrons that are maximally modulated $\left(\eta \sim \pm \eta_{0}\right)$. In other words, the envelope of energy modulation determines the distribution of the secondary microbunches in the phase 


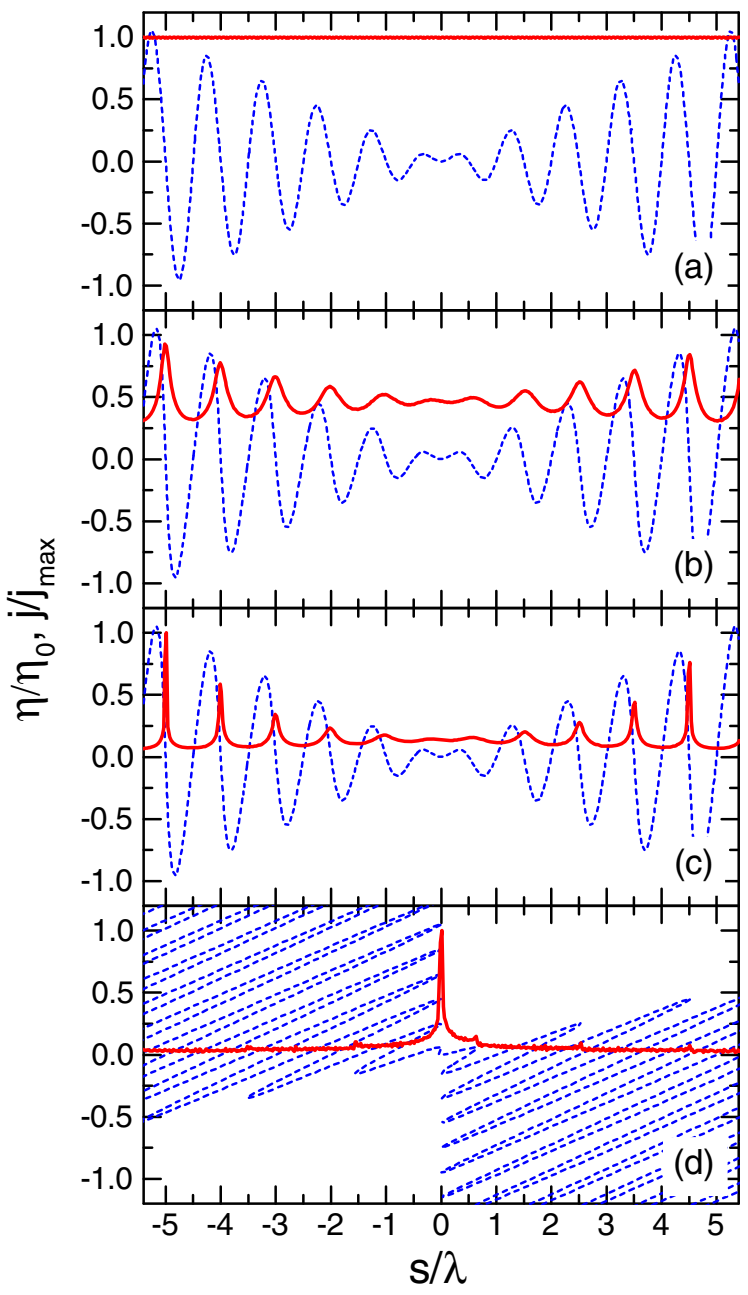

FIG. 2. Same as Fig. 1, but with the modulation amplitude linearly changing with the rate of $\alpha=0.2 \lambda^{-1}$.

space; in this example where the energy modulation amplitude is constant, the secondary microbunches distribute uniformly along $s$.

The above discussion suggests a scheme to take advantage of the secondary microbunches by "modulating the energy modulation." Let us consider the case when the energy modulation linearly changes, namely,

$$
\eta=\eta_{0} \alpha s^{\prime} \sin k s^{\prime}
$$

with $\alpha$ denoting the variation rate. The electron distributions and current profiles calculated in the same manner as done before, are plotted in Figs. 2(a)-2(d), with $\alpha=0.2 \lambda^{-1}$. In this condition, the (normal) microbunches located near $s=$ $\pm 5 \lambda$ are fully compressed with the optimized condition $\hat{r}=$ 1 as found in Fig. 2(c).

What is important is the overcompressed condition (d); because the modulation amplitude is not constant, the energies of the secondary microbunches depend on the initial coordinate $s^{\prime}$ and their motions in the phase space are described as

$$
\begin{gathered}
s \sim s^{\prime} \pm R_{56} \eta_{0} \alpha s^{\prime}, \\
\eta \sim \pm \eta_{0} \alpha s^{\prime} .
\end{gathered}
$$

Substituting the conditions $\alpha=0.2 / \lambda$ and $R_{56}=10 \pi \rho=$ $5 \lambda / \eta_{0}$, we have $(s, \eta) \sim\left(0,-\eta_{0} \alpha s^{\prime}\right)$ and $(s, \eta) \sim\left(2 s^{\prime}, \eta_{0} \alpha s^{\prime}\right)$. The former condition means that half of the secondary microbunches are bundled together at $s=0$, and an isolated current spike is generated as found in Fig. 2(d).

The above result can be generalized as follows. An electron beam with an energy modulation whose amplitude linearly changes with the rate of $\alpha \eta_{0}$ can be effectively compressed by a magnetic chicane with the longitudinal dispersion of $R_{56}=\left(\alpha \eta_{0}\right)^{-1}$.

\section{EXAMPLE}

One example to apply the aforementioned concept is illustrated in Fig. 3, in which the electron beam and seed laser pulse are injected synchronously into the modulator. Although the layout is similar to the seeded FEL, the temporal profile of the seed pulse is different; before injection to the modulator, it is split into two by a half mirror, one of which is delayed by a time delay of $\Delta t$, to be $180^{\circ}$ out of phase with respect to the other. Namely, $\Delta t$ should satisfy the condition $\Delta t=(m+1 / 2) \lambda / c$, where $c$ denotes the speed of light and $m$ is an integer. The two laser beams are then combined and injected into the modulator to interact with the electron beam. Note that the waveform of the combined laser is modulated as schematically illustrated in Fig. 3. If the number of modulator periods is not too large and the slippage effect is not dominant, the energy modulation in the electron beam is similar to the waveform

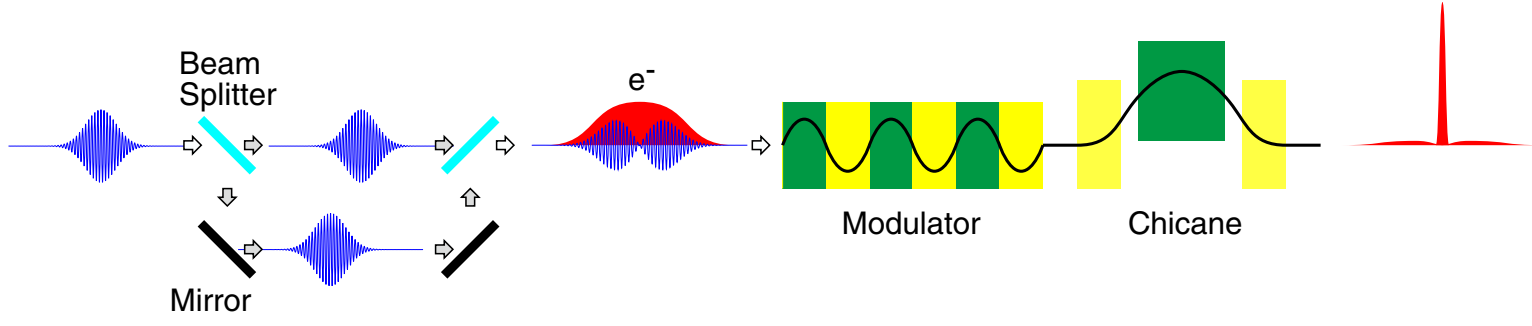

FIG. 3. Schematic illustration to generate an isolated current spike based on the proposed scheme. Note that the subsequent undulators or other devices for specific applications are not shown. 
of the seed pulse, which has a linear variation around the center of the laser pulse. This works to effectively compress the electron beam to generate an isolated current spike, if the magnetic chicane is optimized, which is then used for specific applications such as driving the FEL and generating (coherent or incoherent) synchrotron radiation.

To demonstrate the performance of the proposed scheme, when applied to an FEL driver, we performed numerical simulations assuming that an electron beam with the energy of $2 \mathrm{GeV}$, energy spread of $10^{-4}$, peak current of $2 \mathrm{kA}$, and FWHM bunch length of $200 \mathrm{fs}$ is injected into a modulator with the period length of $120 \mathrm{~mm}$, total length of $0.6 \mathrm{~m}$, and peak field of $1.8 \mathrm{~T}$, to interact with a seed laser having the wavelength of $800 \mathrm{~nm}$, FWHM pulse length of $40 \mathrm{fs}$, and pulse energy of $2 \mathrm{~mJ}$. Before injection into the modulator, the seed laser is split and combined with the temporal delay

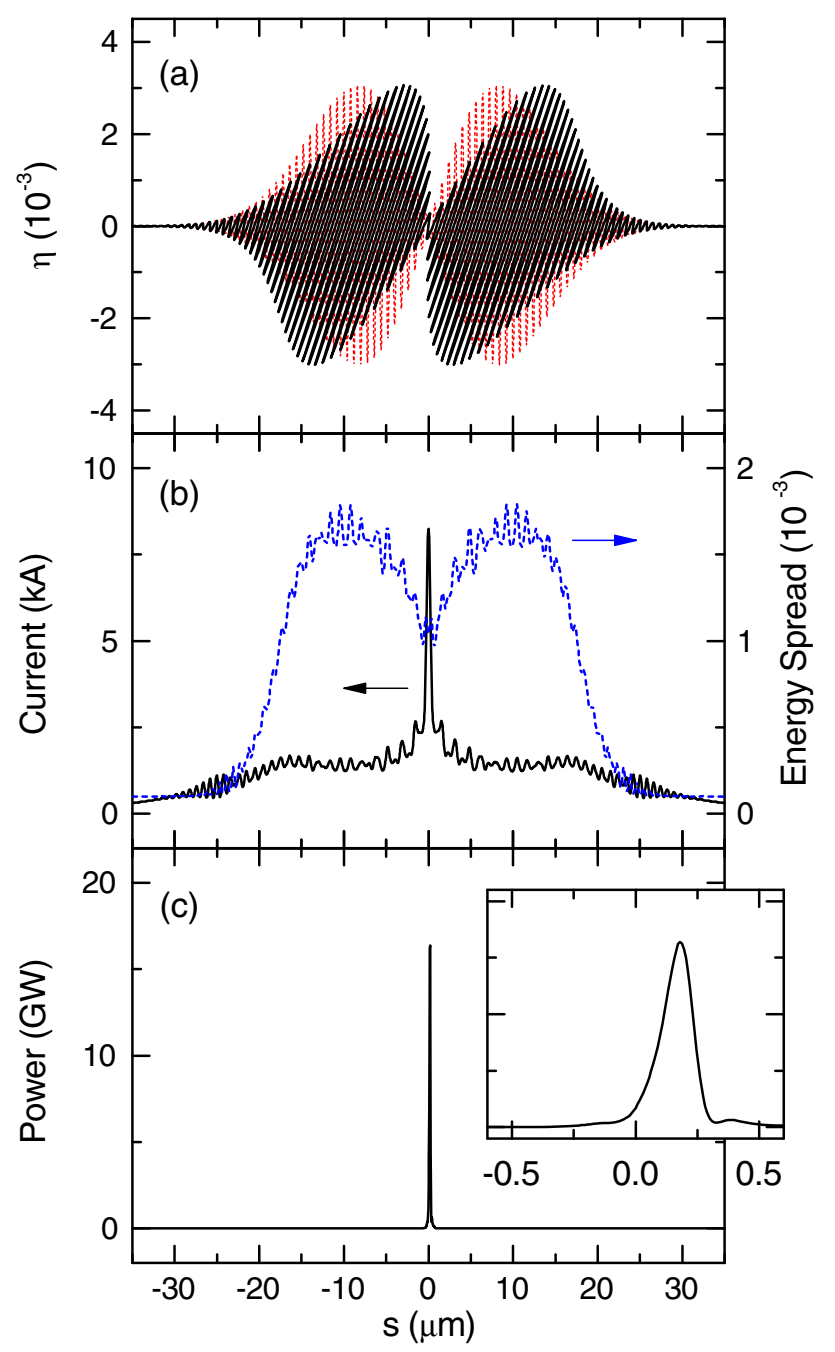

FIG. 4. Results of simulation to demonstrate the performance of the proposed scheme: (a) electron distribution before (solid line) and after (dashed line) the chicane, (b) current profile (solid line) and energy spread (dashed line), and (c) temporal profile of the SASE FEL pulse. of $40 \mathrm{fs}$. After the modulator, the electron beam passes through a magnetic chicane with $R_{56}=0.18 \mathrm{~mm}$ to be compressed with the proposed scheme, and is finally injected into an undulator with the period length of $20 \mathrm{~mm}$, total length of $8 \mathrm{~m}$, and peak field of $1.1 \mathrm{~T}$ to generate an FEL pulse based on the self amplified spontaneous emission (SASE) at the wavelength of $2 \mathrm{~nm}$. Note that the undulator is divided into four segments with $0.5-\mathrm{m}-$ long intersections, where quadrupole magnets are inserted so that the average betatron function is around $4 \mathrm{~m}$.

The numerical simulations have been performed with the FEL simulation code SIMPLEX [20], the results of which are plotted in Figs. 4(a)-4(c).

Figure 4(a) shows the electron distribution in the longitudinal phase space before (dashed line) and after (solid line) the chicane. Note that only the electrons that are initially cold $\left(\eta^{\prime}=0\right)$ are plotted to facilitate visualization, though the effects due to the energy spread are actually taken into account in the simulation. As expected, the energy modulation amplitude linearly changes around the center $s=0$ with the rate of $\alpha \eta_{0}=550 \mathrm{~m}^{-1}$, which is evaluated from the distribution before the chicane (dashed line), and is used to determine the dispersion of the chicane $\left(R_{56}=0.18 \mathrm{~mm}\right)$.

Figure 4(b) shows the current profile (solid line) and slice energy spread (dashed line) along $s$ after the chicane, in which we find that an isolated current spike with the peak current of $8 \mathrm{kA}$ and FWHM bunch length of $0.69 \mu \mathrm{m}$ is generated, though at the expense of the growth of the energy spread $\left(\sim 10^{-3}\right)$. Figure 4 (c) shows the temporal profile of the SASE FEL pulse generated by this electron beam at the exit of the undulator, showing that an attosecond pulse, with the peak power of $16 \mathrm{GW}$ and FWHM pulse length of $0.14 \mu \mathrm{m}$ (470 as), is generated in this example.

Figure 5 shows the spectrum of the SASE FEL pulse computed from the simulation results explained above.

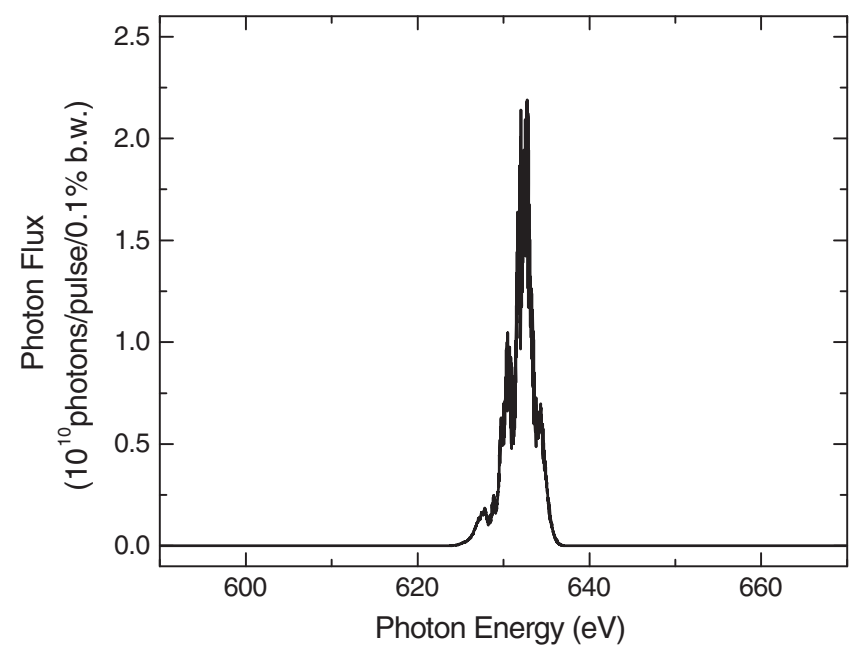

FIG. 5. Spectrum of the SASE FEL pulse retrieved from the simulation results shown in Fig. 4. 
The FWHM bandwidth evaluated by Gaussian fitting is $3.7 \mathrm{eV}$, which roughly corresponds to the bandwidth of a Fourier-transform-limited pulse with the FWHM pulse length of 470 as. This means that the FEL pulse in this example is temporally coherent.

\section{SUMMARY}

In this paper, we proposed a scheme to effectively compress an electron beam with an optical laser, with which the bunch length can potentially be much shorter than what is achieved with the conventional rf technique. We emphasize that the proposed scheme can be easily implemented in existing FEL facilities that are capable of optical laser seeding; what is needed is to insert the splitand-delay optics in the seed laser beamline. It should be mentioned that the compression factor, or the peak current achieved with the proposed scheme, may be lower than that with the THz-radiation scheme, assuming that the $\mathrm{THz}$ radiation is intense enough to induce the energy modulation comparable to that of the optical laser. This is evident from the fact that half of the secondary microbunches do not contribute to the formation of the current spike. As mentioned in the Introduction, however, the energy conversion efficiency of the laser-based $\mathrm{THz}$ source is relatively low for the moment, and thus the energy modulation directly induced by the optical laser is usually much higher. In this regard, the proposed scheme is more feasible, and can be an attractive option to generate extremely short FEL pulses as demonstrated in the numerical example.

\section{ACKNOWLEDGMENTS}

This work was supported by JSPS KAKENHI Grant No. JP18H03691.

[1] W. Ackermann et al., Operation of a free-electron laser from the extreme ultraviolet to the water window, Nat. Photonics 1, 336 (2007).

[2] P. Emma et al., First lasing and operation of an ångstromwavelength free-electron laser, Nat. Photonics 4, 641 (2010).

[3] T. Ishikawa et al., A compact X-ray free-electron laser emitting in the sub-ångström region, Nat. Photonics 6, 540 (2012).

[4] E. Allaria et al., Highly coherent and stable pulses from the FERMI seeded free-electron laser in the extreme ultraviolet, Nat. Photonics 6, 699 (2012).

[5] T. Tschentscher, C. Bressler, J. Grnert, A. Madsen, A. P. Mancuso, M. Meyer, A. Scherz, H. Sinn, and U. Zastrau, Photon beam transport and scientific instruments at the European XFEL, Appl. Sci. 7, 592 (2017).

[6] C. J. Milne et al., SwissFEL: The swiss X-ray free electron laser, Appl. Sci. 7, 720 (2017).
[7] I. S. Ko et al., Construction and commissioning of PALXFEL facility, Appl. Sci. 7, 479 (2017).

[8] R. Akre, D. Dowell, P. Emma, J. Frisch, S. Gilevich, G. Hays, P. Hering, R. Iverson, C. Limborg-Deprey, H. Loos, A. Miahnahri, J. Schmerge, J. Turner, J. Welch, W. White, and J. Wu, Commissioning the Linac Coherent Light Source injector, Phys. Rev. Accel. Beams 11, 030703 (2008).

[9] K. Togawa, T. Hara, and H. Tanaka, Electron-bunch compression using a dynamical nonlinearity correction for a compact X-ray free-electron laser, Phys. Rev. Accel. Beams 12, 080706 (2009).

[10] B. Marchetti, M. Krasilnikov, F. Stephan, and I. Zagorodnov, Compression of a $20 \mathrm{pC}$ e-bunch at the European XFEL for single spike operation, Phys. Procedia 52, 80 (2014), proceedings of the workshop.

[11] S. Huang, Y. Ding, Z. Huang, and J. Qiang, Generation of stable subfemtosecond hard X-ray pulses with optimized nonlinear bunch compression, Phys. Rev. Accel. Beams 17, 120703 (2014).

[12] M. W. Guetg, A. A. Lutman, Y. Ding, T. J. Maxwell, F.-J. Decker, U. Bergmann, and Z. Huang, Generation of HighPower High-Intensity Short X-Ray Free-Electron-Laser Pulses, Phys. Rev. Lett. 120, 014801 (2018).

[13] W. D. Kilpatrick, Criterion for vacuum sparking designed to include both rf and dc, Rev. Sci. Instrum. 28, 824 (1957).

[14] M. C. Thompson, H. Badakov, A. M. Cook, J. B. Rosenzweig, R. Tikhoplav, G. Travish, I. Blumenfeld, M. J. Hogan, R. Ischebeck, N. Kirby, R. Siemann, D. Walz, P. Muggli, A. Scott, and R. B. Yoder, Breakdown Limits on Gigavolt-per-Meter Electron-Beam-Driven Wakefields in Dielectric Structures, Phys. Rev. Lett. 100, 214801 (2008).

[15] M. Dal Forno, V. Dolgashev, G. Bowden, C. Clarke, M. Hogan, D. McCormick, A. Novokhatski, B. Spataro, S. Weathersby, and S. G. Tantawi, rf breakdown tests of mmwave metallic accelerating structures, Phys. Rev. Accel. Beams 19, 011301 (2016).

[16] E. A. Nanni, W. R. Huang, K.-H. Hong, K. Ravi, A. Fallahi, G. Moriena, R. J. Dwayne Miller, and F.X. Kärtner, Terahertz-driven linear electron acceleration, Nat. Commun. 6, 8486 (2015).

[17] L. Zhao, T. Jiang, C. Lu, R. Wang, Z. Wang, P. Zhu, Y. Shi, W. Song, X. Zhu, C. Jing, S. Antipov, D. Xiang, and J. Zhang, Few-femtosecond electron beam with terahertzfrequency wakefield-driven compression, Phys. Rev. Accel. Beams 21, 082801 (2018).

[18] D. Zhang, A. Fallahi, M. Hemmer, X. Wu, M. Fakhari, Y. Hua, H. Cankaya, A.-L. Calendron, L. E. Zapata, N. H. Matlis, and F. X. Kärtner, Segmented terahertz electron accelerator and manipulator (STEAM), Nat. Photonics 12, 336 (2018).

[19] L. H. Yu, Generation of intense uv radiation by subharmonically seeded single-pass free-electron lasers, Phys. Rev. A 44, 5178 (1991).

[20] T. Tanaka, SIMPLEX: simulator and postprocessor for free-electron laser experiments, J. Synchrotron Radiat. 22, 1319 (2015). 